\title{
Medicamentos como fatores de risco para hipertensão arterial secundária: um saber necessário para a enfermagem
}

\author{
Fernanda Furtado da Cunha*, Luciana de Paiva Rêgo**, Andrezza Ozela de Vilhena***
}

\begin{abstract}
*Acadêmica de enfermagem da Universidade do Estado do Pará, ${ }^{* *}$ Enfermeira formada pela Universidade do Estado do Pará, pós-graduanda em enfermagem do trabalho pela Faculdade Metropolitana da Amazônia, ${ }^{* * *}$ Enfermeira, Professora da Universidade do Estado do Pará
\end{abstract}

\section{Resumo}

A doença hipertensiva atinge uma parcela significativa da populaçáo mundial, e uma das causas é o uso indiscriminado de medicamentos prescritos por médicos ou automedicados. Com base nos fatos, este estudo tem como objetivo identificar os fármacos que elevam a pressão arterial no organismo humano, gerando a hipertensão secundária, dando ênfase a seus efeitos nos sistemas pressóricos. Para tal estudo foi realizada uma pesquisa bibliográfica considerando alguns tópicos como: medicamentos que podem desencadear a hipertensão secundária, hábitos de saúde que se tornam agravantes para tal situação, atuação da enfermagem diante da administração de um desses medicamentos e as complicaçóes desta patologia. Com esta pesquisa, concluiu-se que os principais medicamentos causadores da hipertensão secundária são os antidepressivos, cafeína, ciclosporina, contraceptivos orais, anti-inflamatórios não esteroides e eritropoetina. Os profissionais de enfermagem devem ter conhecimento de quais são esse medicamentos e como estes agem no organismo, a fim de evitar complicaçôes tanto para o paciente quanto para sua atuação profissional, uma vez que são os responsáveis pela administração desses fármacos.

Palavras-chave: hipertensão arterial, medicamentos, Enfermagem.

\section{Abstract \\ Medications as risk factors for having secondary hypertension: knowledge needed for nursing}

The hypertensive disease affects a significant portion of world population; one of the causes is the indiscriminate use of drugs under prescription or self-medication. Based on the facts, this study aimed to identify drugs that increase blood pressure in humans, leading to secondary hypertension, emphasizing its effects on the pressure. For this study a literature search was carried out considering some topics, such as products that may trigger secondary hypertension, health habits that will aggravate hypertension, nursing activities before the administration of these drugs and the complications of this pathology. With this research, it was concluded that a major drugs that cause secondary hypertension are antidepressants, caffeine, cyclosporine, 
oral contraceptives, nonsteroidal anti-inflammatory drugs and erythropoietin. Nurses should be aware of which drugs may cause this disease and how they act in the body in order to avoid complications for both the patient and to their professional practice, since they are responsible for the administration of these drugs.

Key-words: hypertension, medicine, nursing.

\section{Resumen}

\section{Medicamentos como factores de riesgo para la hipertensión secundaria: un conocimiento necesario para la enfermería}

La enfermedad hipertensiva afecta a una porción significativa de la población mundial, una de las causas del uso indiscriminado de medicamentos, que a menudo son prescritos por médicos y automedicación. Con base en los hechos, este estudio tiene como objetivo identificar los fármacos que aumentan la presión arterial en los seres humanos, lo que lleva a la hipertensión secundaria, haciendo hincapié en sus efectos sobre la presión. Para este estudio se realizó una búsqueda bibliográfica considerando los siguientes temas: productos que pueden desencadenar la hipertensión secundaria, hábitos de salud que agravan esa situación, actuación de enfermería frente a la administración de estos fármacos y las complicaciones de esa patología. Con esta investigación, se concluyó que los principales medicamentos que causan la hipertensión secundaria son los antidepresivos, la cafeína, ciclosporina, anticonceptivos orales, antiinflamatorios y la eritropoyetina. Los enfermeros deben ser conscientes de las indicaciones de los medicamentos y cómo actúan en el cuerpo con el fin de evitar complicaciones para el paciente y su práctica profesional, ya que son responsables por la administración de estos fármacos.

Palabras-clave: hipertensión, drogas, enfermería.

\section{Introdução}

A doença hipertensiva é caracterizada por ser uma doença quase sempre assintomática, o que justifica a sinonímia de "doença silenciosa", portanto na maioria dos casos o seu diagnóstico é tardio. O Ministério da Saúde estima que 35\% da população acima de 40 anos tenha hipertensão arterial [1].

A doença hipertensiva é uma síndrome cardiovascular progressiva, crônica não transmissível, surgindo a partir de complexos e aspectos etiológicos, tendo como característica a elevação da pressão arterial e com anormalidade no funcionamento de estruturas cardíacas e vasculares com lesōes em órgãos como coração, cérebro, rins, artérias e outros órgãos, levando a morbidades e mortalidades [2].

A doença hipertensiva resulta do aumento do tônus do músculo liso vascular periférico, que leva ao aumento da resistência arteriolar e a redução da capacitância do sistema nervoso, sendo definida como Pressão Sanguínea Sistólica (PSS) sustentada maior que $140 \mathrm{mmHg}$ e/ou uma Pressão Sanguínea Diastólica (PSD) superior a $90 \mathrm{mmHg}$ [3].

Logo a doença hipertensiva se trata de um importante problema de saúde pública que deve ser abordado pelos profissionais de saúde para melhor atuação, diagnóstico, prevenção e tratamento.
Sabendo-se que certos fármacos têm a propriedade de elevar a pressão arterial, faz-se necessária uma história clínica minuciosa, considerando o histórico clínico do paciente. O manuseio adequado é fundamental para evitar complicaçóes mais severas, como desenvolvimento de crises hipertensivas, infarto agudo do miocárdio, acidente vascular cerebral e até mesmo morte [1].

\section{Material e métodos}

O presente estudo tem como proposta identificar as causas da doença hipertensiva secundária relacionada ao uso de fármacos que favorecem o aumento dos níveis pressóricos no organismo humano. O estudo é descritivo e foi desenvolvido a partir de uma pesquisa bibliográfica, com abordagem qualitativa. Nesse contexto, considerou-se os componentes da situaçáo em estudo e suas interações, buscando uma visão integrada. Para a análise e interpretação dos resultados foram considerados os conhecimentos teóricos sobre o assunto, bem como as relaçóes existentes entre as variáveis envolvidas a partir dos dados pesquisados. No processo, também, foi discutido como os achados decorrentes do trabalho podem influenciar a vida das pessoas envolvidas e na saúde pública, e 
ressalta como os dados obtidos podem ser usados para melhorar o cotidiano.

\section{Resultados e discussão}

De acordo com a pesquisa desenvolvida, pode-se ter conhecimento dos vários fármacos que podem desencadear a doença hipertensiva, dentre eles estão: 1) Antidepressivos - conhecidos como tricíclicos e tetracíclicos juntamente com os inibidores da monoaminoxidase (IMAOs) são considerados antidepressivos clássicos. Os principais efeitos das drogas tricíclicas e tetracíclicas são exercidos sobre o sistema nervoso central, embora efeitos anticolinérgicos das drogas produzam uma faixa diversificada de efeitos adversos mediados pelo sistema nervoso autônomo, além dos efeitos significativos no sistema cardiovascular.

Em dosagens terapêuticas, as drogas são classificadas como drogas antiarrítmicas do tipo 1A, já que terminam com a fibrilação ventricular e podem aumentar o suprimento sanguíneo colateral para um coração isquêmico. Entre os antidepressivos temos: Imipramina, Desipramina, Trimipramina, Amitriptilina, Doxepina, Maprotilina, dentre outros. Estas podem causar taquicardia, aplainamento das ondas $\mathrm{T}$, prolongamento de intervalos QT e depressão dos segmentos ST no registro eletrocardiográfico. A imipramina tem efeito tipo quinidina em níveis plásmicos terapêuticos e pode reduzir o número de extrassístoles. E ainda em casos de interaçóes medicamentosas, os antidepressivos tricíclicos e tetracíclicos bloqueiam a recaptação neuronal da guanetidina, necessária para a atividade anti-hipertensiva.

Os efeitos anti-hipertensivos dos antagonistas de receptores beta-adrenergicos, por exemplo, propranolo e da clonidina também podem ser bloqueados pelas drogas tricíclicas e tetracíclicas [4]. Um dos efeitos adversos desta medicação é a crise hipertensiva, ainda que de ocorrência rara, produzida quando esta droga é usada em associação com uma droga simpatomimética (fenilpropanolamina e dextrometorfano, encontrados nos descongestionantes nasais adquiridos sem prescrição médica e antitussígenos) ou alimentos e/ou bebidas que contenham tiramina (como queijos, vinhos, licores), ou ainda em alimentos conservados (molho de soja, caviar, uvas passas etc.).

A crise hipertensiva relacionada aos IMAOs se manifesta por cefaleia pulsátil acompanhada de elevaçóes graves da pressão arterial. O tratamento da crise compreende o uso de clorpromazina, inicialmente, e o paciente deve ser orientado a procurar auxílio médico imediato para controle da pressão arterial.

A buspirona, um alfa agonista do receptor tipo $1 \mathrm{da}$ serotonina também tem sido relacionada a um aumento dos níveis pressóricos decorrente de seu metabolito 1,2-pirimidinil-piperazina, que é um antagonista do receptor alfa2-adrenergico. Esta elevação de pressão é descrita não apenas como a buspirona, mas com outros agonistas da serotonina [5].

A cafeína, conhecida como a droga mais consumida no mundo, está presente em diversos alimentos como cacau, refrigerantes, chocolate, chá mate, chás em geral, medicamentos do tipo analgésicos e antigripais. Presente em mais de 60 espécies de plantas, a cafeína é a droga psicoativa mais consumida. Essa substância pertence ao grupo de compostos das metilxantinas, no qual se inclui também o chá. As xantinas são substancias capazes de estimular o sistema nervoso, produzindo certo estado de alerta de curta duração. As xantinas são bases nitrogenadas da mesma classe (alcalóides) em que se incluem a atropina, cocaína, efedrina, morfina, quinina, nicotina, e varias outras, todas relacionadas à grande variedade de açóes fisiológicas. A xantina por sua vez é uma substancia química derivada da purina, é uma dioxipurina estruturalmente relacionada com o ácido úrico. A ligação da adenosina, um neurotransmissor natural, aos seus receptores, diminui a atividade neural, dilata os vasos sanguíneos, entre outros. Com isso, a cafeína se liga aos receptores da adenosina e impede a ação da mesma sobre o SNC [5].

Muitos medicamentos contra cefaleias, tal como a aspirina forte, contém cafeína. Ela irá contrair os vasos sanguíneos e aliviar a dor. Com o aumento da atividade neural, a glândula pituitária reage como se algum tipo de emergência estivesse ocorrendo, e libera grandes quantidades de adrenalina, causando uma série de efeitos no corpo humano, como taquicardia, aumento da pressão arterial, abertura dos tubos respiratórios (por isso muitos medicamentos contra asma contém cafeína), aumento do metabolismo e contração dos músculos, entre outros. A cafeína também aumenta a concentraçáo de dopamina no sangue por diminuir a recaptação desta no SNC. A dopamina também é um neurotransmissor, relacionado com o prazer, 
e suspeita-se que seja justamente este aumento dos níveis de dopamina que leve ao vício da cafeína. Essa substância, em curto prazo, impede o bloqueio e a recepção de adenosina, dando mais disposição, pois causa liberação de adrenalina, e lhe faz "sentir melhor", pois manipula a produção de dopamina [6].

A cafeína resulta numa estimulação dos sistemas envolvidos, aumentando tanto a liberaçáo de norepinefrina como a taxa de ativação espontânea dos neurônios noradrenergicos. Entre os efeitos autonômicos estimulantes da cafeína como antagonista da adenosina observa-se a estimulação cardíaca, aumento da pressão arterial, redução da mobilidade intestinal enfim, produz-se um clássico estado de estimulação simpática, tal como se houvesse uma atitude e estresse onde as reservas corporais se mobilizariam. Na hipersensibilidade a cafeína sente-se inquieta, agitada, com um discreto mal-estar e ansiedade. A seguir ocorre taquicardia, sensação de zumbido no ouvido e distúrbios visuais parecendo faíscas no ar. A musculatura torna-se tensa e tremula, podem ocorrer palpitaçóes devido ao surgimento de extra-sístoles no coraçáo [7].

A ciclosporina é uma droga imunossupressora, porém sem qualquer efeito sobre a reação inflamatória aguda. Foi descoberta por Borel e colaboradores em 1976 durante uma triagem de produtos fúngicos, foi isolada do fungo Tolypocladium inflantum, habitante do solo. As ciclosporinas são indicadas para a redução de incidência de rejeição de enxerto em transplantes de órgáos e para variadas doenças autoimunes. A principal açáo da ciclosporina consiste em ocorrer uma diminuição da proliferação clonal de células $T$, primariamente ao inibir a liberação de interleucina- 2 e, possivelmente, também, ao reduzir a expressão dos receptores da interleucina-2. Esta diminuiçáo ocorre devido à ciclosporina ligar-se a uma proteína citosólica, denominada imunofilinas [8].

O complexo fármaco/imunofilin liga-se a calcineurina e a inibe, interferindo assim na ativação das células Th e na produçáo IL-2. O resultado do estudo geral com o tema "Efeitos cardiovasculares num modelo experimental", realizados em ratos Wistar, machos, durante sete (7) semanas com CsA (Sandimmun Neoral) dissolvida em suco de laranja, nas doses de $5 \mathrm{mg} / \mathrm{kg} /$ dia em um grupo de ratos e $30 \mathrm{mg} / \mathrm{kg} /$ dia em outro grupo. Na segunda semana os dois grupos já apresentavam hipertensão arterial. Seguindo o estudo, concluiu-se que os resultados obtidos sugerem que os efeitos cardiovasculares da CsA no rato Wistar sáo precoces e se caracterizam por HTA, e após sete semanas de tratamento com doses elevadas correspondente a Cmax humana por cardiopatia isquêmica e taquicardia, não apresentando hipertrofia ventricular esquerda. A dose inferior 5 $\mathrm{mg} / \mathrm{kg} /$ dia induz menores lesóes cardíacas, aumentando, no entanto, a pressão arterial diferencial. A função renal, para ambas as doses de ciclosporinas, apresentou-se sem alteraçóes significativas, o que os levou a sugerir que estas alteraçóes, se existirem, serão posteriores as alteraçóes cardiovasculares, nomeadamente a HTA [9].

A problemática das ciclosporinas está nos seus efeitos adversos, tendo como efeito mais comum nefrotoxicidade, hepatotoxicidade e hipertensão. Os menos relevantes incluem anorexia, letargia, hirsutismo, tremor, parestesia, hipertrofia gengival e distúrbios gastrointestinais. O que leva o paciente a apresentar um quadro de doença hipertensiva secundaria é a vasoconstrição renal intensa, ativação do sistema nervoso simpático, inibição do óxido nítrico, aumento do influxo de cálcio, retençáo de volume com supressão do sistema renina-angiotensina-aldosterna, dentre outros [10].

Os contraceptivos orais, desde sua introdução no início dos anos 60, começaram a ser publicados casos na literatura que relacionavam o uso de contraceptivos orais (CO) a aumentos dos níveis pressóricos. A presença de hipertensão arterial leve é habitualmente uma contraindicação relativa para o uso de contraceptivos orais, e enquanto nas pacientes com hipertensão arterial mais grave, com fatores de risco adicionais para doença cardiovascular ou quando houver lesão em órgãos-alvo, é considerada uma contraindicaçáo. $\mathrm{Na}$ maioria das mulheres o uso de contraceptivos orais (CO) causa ligeira elevação dos níveis pressóricos. A magnitude da elevação pressórica varia de acordo com a população avaliada e a dose do contraceptivo, variando de 3-6 mmHg na sistólica e 2-4 $\mathrm{mmHg}$ na diastólica, quando a dose de estrógeno variava de 30-35 Ug de estrógeno 3,4. Em alguns casos, entretanto, o uso de $\mathrm{CO}$ pode desencadear o desenvolvimento da hipertensão arterial que se mostra reversível com sua suspensão [4].

O mecanismo pelo qual os $\mathrm{CO}$ causa elevação da pressão arterial deve-se a retenção de sódio pelo estrógeno e parte da progesterona sintética contida nos contraceptivos. O diagnóstico de hipertensão causada por $\mathrm{CO}$ resulta da normalização dos níveis pressóricos a partir da descontinuaçáo de sua administração. Esta normalizaçáo é vista em média 3 
meses após a suspensão do $\mathrm{CO}$, embora em algumas pacientes isto possa ocorrer em até 6 meses. Em alguns destes casos, recomenda-se o monitoramento da pressão arterial com maior frequência de modo a viabilizar o uso dos $\mathrm{CO}$ quando náo houver outra alternativa.

Entre as drogas anti-hipertensivas recomendadas para uso na vigência de $\mathrm{CO}$, estâo os diuréticos que atuam sobre a retenção de sódio. Já com relação à terapia de reposição hormonal o uso de estrógenos ou estrógenos combinados com progestágenos não constitui um contra, pois estas substâncias são, em geral, administradas em baixas doses, podendo ocorrer inclusive uma ligeira redução dos níveis pressóricos. Mesmo assim, recomenda-se o monitoramento da pressáo arterial, pois em raros casos observou-se elevação da pressão [6].

Os antiflamatórios não esteroides (AINEs) (antipiréticos e analgésicos) estão entre as drogas mais prescritas no Brasil [1]. Dada a alta prevalência da hipertensão arterial, é muito frequente o uso concomitante de AINEs e anti-hipertensivos no mesmo paciente, assim como o surgimento de interaçóes medicamentosas clinicamente significativas entre tais classes de fármacos. Há estudos que têm como enfoque o efeito dos AINEs sobre a pressão arterial, gerando a diminuição da eficácia dos medicamentos anti-hipertensivos. Os AINEs não-seletivos agem sobre a COX1 e COX2 que principalmente se envolvem em interaçóes potencias com os anti-hipertensivos como o ibuprofeno, naproxeno, piroxicam, indometacina, diclofenaco e o ácido acetilsalicitico [11].

Outros, inclusive analgésicos e antipiréticos, como a dipirona e o paracetamol, também podem interferir na açáo dos anti-hipertensivos. O principal mecanismo que envolve a elevação da pressão arterial pelos AINEs é a inibição da enzima COX. Tal inibição gera a redução sistêmica e renal da síntese de prostaglandinas (PGs). No rim, a inibição da enzima COX gera diminuição das taxas de PGI e PGE2. Tais prostaglandinas agem mantendo a homeostasia renal por regulação da reabsorção de sódio e água, mediando o tônus vascular e a liberaçáo de renina.

O uso de AINEs é limitado e é motivo de precaução por desenvolverem hipertensão, edema, doença cardíaca congestiva, acidente vascular cerebral em uma proporção significativa de pacientes. $\mathrm{O}$ efeito dos AINEs na instabilidade da pressão arterial é clinicamente relevante tanto em pacientes em tratamento anti-hipertensivo como em aqueles sem tratamento. Todos os AINEs podem antagonizar a terapia anti-hipertensiva, seja parcial ou totalmente. Dessa forma, podem aumentar a morbidade relacionada à hipertensão arterial.

$\mathrm{O}$ efeito na pressáo arterial pode variar de nenhum até crises hipertensivas. $\mathrm{O}$ risco é maior em idosos, afrodescendentes e naqueles com hipertensão arterial com renina baixa. Recomenda-se que pacientes com doença hipertensiva controlada com medicamentos sejam submetidos a monitoramento cuidadoso da pressão arterial e da funçáo renal após o início da terapia com AINEs e que se avaliem os benefícios do tratamento em relação aos riscos. Em caso de perceber qualquer dano ao paciente, deve-se interromper a terapia do anti-inflamatório, mudar o agente anti-hipertensivo ou alterar a dose deste último. Entretanto estudos atuais demonstram que a dipirona, quando administrada intravenosamente, pode causar diminuição da pressão arterial por mecanismos ainda não muito claros, acredita-se, porém, que seja por inibição de canais de potássio dependentes da voltagem no músculo liso vascular. Portanto, sendo a dipirona um fármaco pouco estudado, sua ação na hemodinâmica ainda é controversa [7].

O diclofenaco é considerado o segundo AINE com maior risco de desenvolver eventos cardiovasculares (infarto do miocárdio) em pacientes hipertensos, devido, principalmente, ao aumento da pressão arterial. Já o AAS é relacionado ao aumento dos níveis pressóricos quando em uso crônico, sobretudo no paciente idoso.

Os AINEs associados a outros fármacos podem antagonizar o efeito dos anti-hipertensivos, tal fato pode ser visto com preocupação, pois pode haver um "sinergismo" no antagonismo gerado por tais drogas no efeito dos anti-hipertensivos. Todos os AINEs parecem elevar a pressão arterial média e antagonizar os efeitos hipotensores dos anti-hipertensivos, incluindo os analgésicos e antipiréticos como a dipirona e o paracetamol [12].

A eritropoetina (EPO) é atualmente utilizada no tratamento da anemia de pacientes com doença renal crônica, pode piorar a hipertensão e aumentar a necessidade de drogas anti-hipertensivas. Este efeito, entretanto, não é observado em pacientes recebendo eritropoetina, sugerindo que a doença renal crônica pode conferir uma susceptibilidade particular a ação hipertensiva da eritropoetina. Quanto mais intensa a anemia e mais rápida a sua correção, maior a incidência da hipertensão induzida 
pela eritropoetina recombinante humana. Desde o artigo de Eschbach, que empregou EPO pela primeira vez em humanos em programa de hemodiálise, já foi verificado o advento de hipertensão arterial ou o agravamento de quadro hipertensivo pré-existente, inclusive com descriçóes de quadros graves de encefalopatia hipertensiva. A correção da anemia com eritropoetina está associada com o agravamento ou surgimento de quadro hipertensivo em cerca de $20 \%$ dos pacientes. A monitorizaçáo e o controle dos níveis pressóricos devem ser feitos de maneira rigorosa em todas as situaçóes em que se utiliza a eritropoetina recombinante humana, no sentido de evitarmos o agravamento de doenças vasculares presentes em grande parte dos pacientes portadores de insuficiência renal crônica e o advento de encefalopatia hipertensiva, que muitas vezes pode ser acompanhado de crise convulsiva. Os pacientes em tratamento conversador merecem atenção adicional, pois a doença hipertensiva pode acelerar o quadro de progresso da doença renal. Deve-se manter o controle adequado do volume circulante e dos agentes anti-hipertensivos. Em alguns casos, a dose de eritropoetina pode ser reduzida e a via de administração modificada, de endovenosa para subcutânea [14].

\section{Conclusão}

Os resultados levantados a partir deste estudo enfatizaram a importância do conhecimento técnico que os profissionais de saúde devem adquirir para uma melhor intervençáo no processo de tratamento do paciente. Com isto, verificou-se que os medicamentos possuem efeitos adversos que tem que ser levados em consideraçáo durante a prescrição médica, assim como as interações medicamentosas, que podem ocasionar uma hipertensão arterial secundária. Com base nisto, a enfermagem deve levar educaçáo à comunidade quanto ao perigo da automedicação, assim como ao realizar as consultas de enfermagem estar atento a história de saúde do paciente, os medicamentos em uso, alergias, e todas as informaçóes pertinentes ao cuidado, para assim estar orientando, educando e evitando maiores complicaçóes.

\section{Referências}

1. Ministério da saúde. Hipertensão: um mal que pode ser evitado. [citado 2011 Ago 12]. Disponível em URL: http://portal.saude.gov.br/portal/saude/area.

2. Savage J. Tudo sobre hipertensáo arterial. 1a ed. São Paulo: Andrei; 2000.

3. Póvoa R. Bombig MTN. Cardiopatia Hipertensiva. $1^{\text {a }}$ ed. São Paulo: Atheneu; 2011.

4. Plavnik FL. Hipertensão arterial induzida por drogas: como detectar e tratar. Rev Bras Hipert 2002;9:185-91.

5. Kaplan HI, Sadock BJ, Grebb JA. Compêndio de Psiquiatria: Ciência do comportamento e Psiquiatria Clínica. 7a. ed. Porto Alegre: Artes Médicas; 1997.

6. Póvoa R. Hipertensão arterial a prática clínica. $2^{\mathrm{a}}$. ed. São Paulo: Atheneu; 2007.

7. Rang HP, Dale MM, Ritte JM. Farmacologia. $4^{\mathrm{a}}$. ed. Rio de Janeiro: Guanabara; 2001.

8. Page C. Farmacologia integrada. 2a. Ed. São Paulo: Manole; 2004.

9. Tavares P, Reis F, Ribeiro CAF, Teixeira F. Efeitos cardiovasculares da ciclosporina num modelo experimental. Revista Portuguesa de Cardiologia 2002;21(2):141-55.

10. Kester M. Farmacologia. 1a . ed. São Paulo: Elsevier; 2008.

11. Ribeiro A. Atualização em hipertensão arterial. São Paulo: Atheneu; 2007.

12. Pimenta E, Calhoun DA, Oparil S. Mecanismos e tratamentos da hipertensáo arterial refratária. Arq Bras Cardiol 2007;88(6): 683-92.

13. Abensur $\mathrm{H}$. Reaçôes adversas referentes a eritropoetina. J Bras Nefrol 2000;22(5):39-41.

14. Asperheim M. Farmacologia para Enfermagem. 11 a ed. São Paulo: Elsevier; 2009. 